\title{
Numerical precision of the solution to the running-coupling Balitsky-Kovchegov equation
}

\author{
Marek Matas ${ }^{1, a}$, Jan Cepila ${ }^{1}$, and Jesus Guillermo Contreras Nuno ${ }^{1}$ \\ ${ }^{1}$ Faculty of Nuclear Sciences and Physical Engineering, Czech Technical University in Prague, Prague, \\ Czech Republic
}

\begin{abstract}
We use the running coupling Balitsky-Kovchegov (rcBK) equation to study the rapidity dependence of saturation in inclusive HERA data and we discuss the behaviour of its numerical solution. The rcBK equation has been solved using Runge-Kutta methods. The influence of the parameters implicit in the numerical evolution has been studied. They include, among others, the order of the Runge-Kutta evolution, the size of the different grids and the step in the numerical evolution. Some suggestions on the minimum value of these parameters are put forward.
\end{abstract}

\section{Introduction}

One of the open questions in particle physics is particle production in the high-energy limit of QCD. Evolution equations such as BFKL [1-5] are used to describe the gluon density of the hadron in highenergy collisions. The BFKL evolution equation predicts the emergence of new partons as the energy of the collision increases and in this approach, the gluon density is not bound by unitarity restrictions. The number of partons may be reduced by saturation effects caused by recombination processes inside the hadron. An extension of the BFKL equation was found by Balitsky [6] and Kovchegov [7] (BK) resulting in an equation that generates dynamical balance between radiation and recombination processes driven by a saturation scale. The integro-differential BK evolution equation [8-10] considered in this work, and denoted by rcBK in the following, includes a running-coupling kernel that takes into account two-loop processes and assumes an impact-parameter independent solution [11]. It was derived from the JIMWLK evolution equations in the limit of large number of colors by Kovchegov $[7,12]$.

Using numerical methods one can solve the rcBK equation [8-10, 13] and calculate a prediction for the proton structure of DIS that accounts for recombination processes within the hadron. Numerical methods used to compute the rcBK solution need to be studied and an optimal setup regarding precision and speed of computation has to be tested in order to estimate the uncertainty coming from the non-analytic solution of rcBK equation.

\section{The rcBK evolution equation}

The rcBK evolution equation with running coupling kernel reads [14].

\footnotetext{
a e-mail: matas.marek1@gmail.com
} 


$$
\frac{\partial N(r, Y)}{\partial Y}=\int d \overrightarrow{r_{1}} K^{r u n}\left(\vec{r}, \overrightarrow{r_{1}}, \overrightarrow{r_{2}}\right)\left(N\left(\overrightarrow{r_{1}}, Y\right)+N\left(\overrightarrow{r_{2}}, Y\right)-N(\vec{r}, Y)-N\left(\overrightarrow{r_{1}}, Y\right) N\left(\overrightarrow{r_{2}}, Y\right)\right),
$$

where $K^{r u n}\left(\vec{r}, \overrightarrow{r_{1}}, \overrightarrow{r_{2}}\right)$ can be expressed as [15]

$$
K^{r u n}\left(\vec{r}, \overrightarrow{r_{1}}, \overrightarrow{r_{2}}\right)=\frac{N_{c} \alpha_{s}\left(r^{2}\right)}{2 \pi^{2}}\left(\frac{r^{2}}{r_{1}^{2} r_{2}^{2}}+\frac{1}{r_{1}^{2}}\left(\frac{\alpha_{s}\left(r_{1}^{2}\right)}{\alpha_{s}\left(r_{2}^{2}\right)}-1\right)+\frac{1}{r_{2}^{2}}\left(\frac{\alpha_{s}\left(r_{2}^{2}\right)}{\alpha_{s}\left(r_{1}^{2}\right)}-1\right)\right),
$$

$\overrightarrow{r_{2}}=\vec{r}-\overrightarrow{r_{1}}$ and $Y=\ln \frac{x_{0}}{x}$. If we disregard the last term in Eq.1, we obtain a linear equation that can be shown to be equivalent to the BFKL evolution equation [16]. The running coupling depends on the number of considered quark flavors, in this work we use $n_{f}=3$

$$
\alpha_{s, n_{f}}\left(r^{2}\right)=\frac{4 \pi}{\beta_{0, n_{f}} \ln \left(\frac{4 C^{2}}{r^{2} \Lambda_{n_{f}}^{2}}\right)},
$$

where

$$
\beta_{0, n_{f}}=11-\frac{2}{3} n_{f}
$$

The constant $C^{2}$ is the uncertainty coming from the Fourier transformation and is usually fitted to data [10], $\Lambda_{n_{f}}^{2}$ is called the scale parameter and its value depends on the value of $n_{f}$ (we set it to $\Lambda_{n_{f}}=0.241 \mathrm{GeV}$ ). Since all dipole sizes are accounted for in the rcBK evolution equation, coupling needs to be reduced after a certain value is reached, so that the maximal value of the coupling constant would not exceed a set limit [10] (in our case $\alpha_{s}=0.7$ ).

We used the MV initial condition [10] with parameters $C=2.52, \Lambda_{Q C D}=0.241 \mathrm{GeV}, \gamma=1.135$, $Q_{s 0}^{2}=0.165 \mathrm{GeV}^{2}, N_{c}=3[10]$,

$$
N^{M V}\left(r, x=x_{0}\right)=1-\exp \left(-\frac{\left(r^{2} Q_{s 0}^{2}\right)^{\gamma}}{4} \ln \left(\frac{1}{r \Lambda_{Q C D}}+e\right)\right),
$$

where $e$ is the elementary charge.

\section{Solving the rcBK evolution equation}

The rcBK equation does not have an analytic solution, so it has to be solved numerically [8-10]. A usual way of solving this equation involves dividing the integral over $r_{1}$ into a logarithmic grid with a constant step, then one uses a Runge-Kutta method for solving the differential equation, the Simpson method for numerical integration and linear interpolation for acquiring values of $N(r)$ for an arbitrary point.

The parameters of the numerical computation have been varied and their influence to the resulting scattering amplitude was studied. The proportional change $D(r, Y)$ was computed as

$$
D(r, Y)=\frac{\left|N_{\text {orig }}(r, Y)-N_{\text {new }}(r, Y)\right|}{N_{\text {orig }}(r, Y)} .
$$

The initial values for the computation were 25 steps over one order of magnitude of the dipole size $r$ over the interval $\left[10^{-7}, 10^{2}\right], 10$ steps over the interval of $[0 ; \pi]$ in $\theta$, which is the angle between $r$ and $r_{1}$, and a Runge-Kutta method of fourth order with step of 0.01 in rapidity. 
In Fig. 1 we can see the integrand for the structure function $F_{2}$ (see for example Eq. 9 in [13]) and its dependence on $r$ for $Y=2$ and $Y=10$. We can see that the interval that contributes the most to the total value of $F_{2}$ is at about $r \sim(0.1,20) \mathrm{GeV}^{-1}$. The highest precision needs to be obtained namely for these values of $r$.
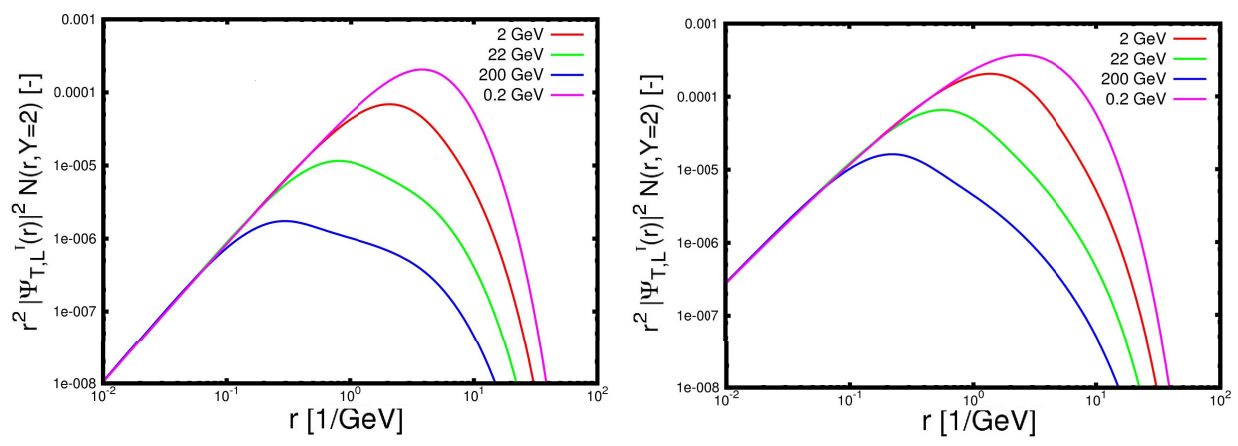

Figure 1. Integrand for the structure function as a function of $r$ for $Y=2$ (left) and $Y=10$ (right). Each line corresponds to a different value of the photon virtuality $Q^{2}$.

A significant decrease in the running time can be also achieved by modifying the Runge-Kutta method. Since the rcBK evolution equation does not explicitly depend on rapidity, we can split the integration into three terms and then obtain all Runge-Kutta coefficients as their linear combination without the need to recompute the integration itself. The three terms stand as follows [13]

$$
\begin{aligned}
& I_{0}=\int d \overrightarrow{r_{1}} K^{r u n}\left(\overrightarrow{r_{1}}, \overrightarrow{r_{2}}, \vec{r}\right) \\
& I_{1}=\int d \overrightarrow{r_{1}} K^{r u n}\left(\overrightarrow{r_{1}}, \overrightarrow{r_{2}}, \vec{r}\right)\left(N\left(Y, \overrightarrow{r_{1}}\right)+N\left(Y, \overrightarrow{r_{2}}\right)\right) \\
& I_{2}=\int d \overrightarrow{r_{1}} K^{r u n}\left(\overrightarrow{r_{1}}, \overrightarrow{r_{2}}, \vec{r}\right)\left(N\left(Y, \overrightarrow{r_{1}}\right) N\left(Y, \overrightarrow{r_{2}}\right)\right)
\end{aligned}
$$

and the Runge-Kutta coefficients can be expressed as

$$
\begin{gathered}
k_{1}=I_{1}-I_{2}-I_{0} \cdot N(r, Y) \\
k_{2}=k_{1}+\frac{1}{2} h k_{1} I_{0}-\frac{1}{2} h k_{1} I_{1}-\frac{1}{4} h^{2} k_{1}^{2} I_{0} \\
k_{3}=k_{1}+\frac{1}{2} h k_{2} I_{0}-\frac{1}{2} h k_{2} I_{1}-\frac{1}{4} h^{2} k_{2}^{2} I_{0} \\
k_{4}=k_{1}+\frac{1}{2} h k_{3} I_{0}-\frac{1}{2} h k_{3} I_{1}-\frac{1}{4} h^{2} k_{3}^{2} I_{0}
\end{gathered}
$$



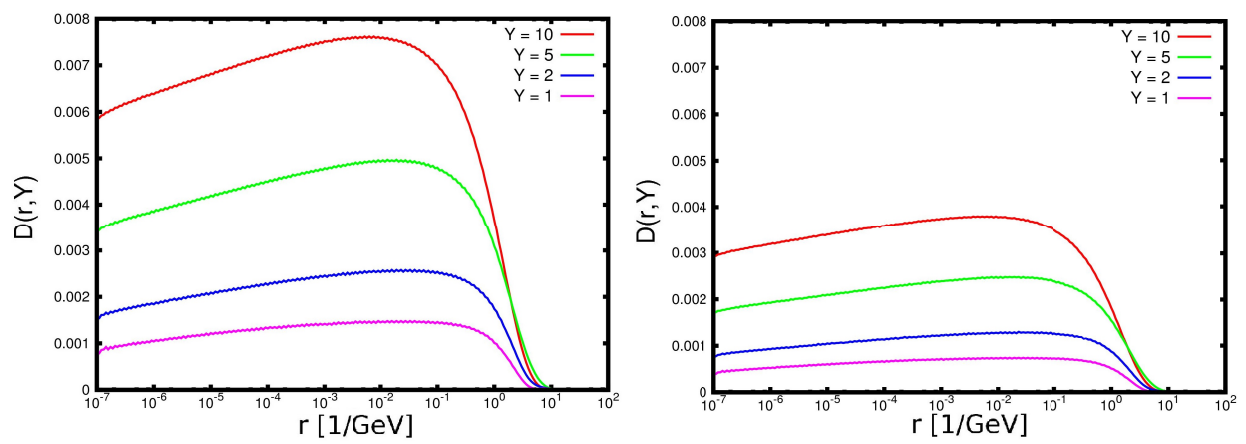

Figure 2. A variation of the Runge-Kutta method. Left - Runge-Kutta method of first order compared to the second, right - second order compared to the fourth.
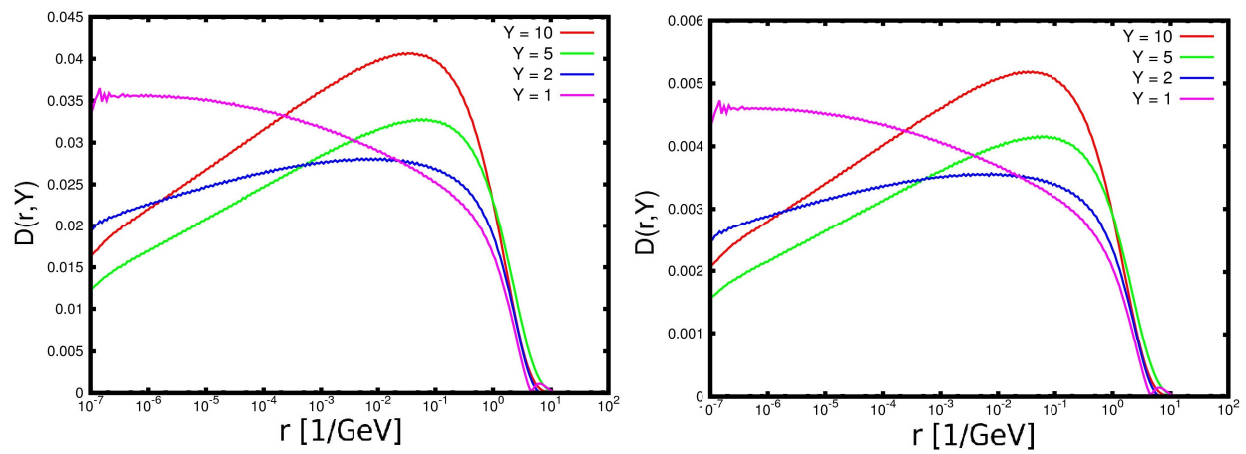

Figure 3. A variation of the rapidity step. Left - step of 0.05 compared to 0.01 , right - step of 0.01 compared to 0.005 .

\section{Results}

There is less than $0.7 \%$ difference in the rcBK solution when comparing first order Runge-Kutta method to the second order up to $Y=10$ and less than $0.4 \%$ for comparing second order to fourth. Therefore, there is less than $1 \%$ uncertainty coming from the choice of the Runge-Kutta method and the uncertainty rises with rapidity. Considering the step in rapidity for the Runge-Kutta method, it can be seen that the difference between the step of 0.5 to the step of 0.1 is up to $4 \%$ and the biggest difference is in the region where the integrand dominates. Therefore it can be presumed that the uncertainty will also rise with rapidity. The difference between the step of 0.1 to the step of 0.05 is less than approximately $0.5 \%$.

When the variation of the number of steps for the magnitude of $r_{1}$ is done from 10 steps to 25 steps per order of magnitude of $r_{1}$, the difference quickly rises with rapidity up to $2.5 \%$ at $Y=10$. Using 50 steps instead of 25 steps does not yield any considerable difference for all rapidities. The results at low values of $r$ have small significance since their contribution to the final structure function is negligible as shown in Fig. 1. 

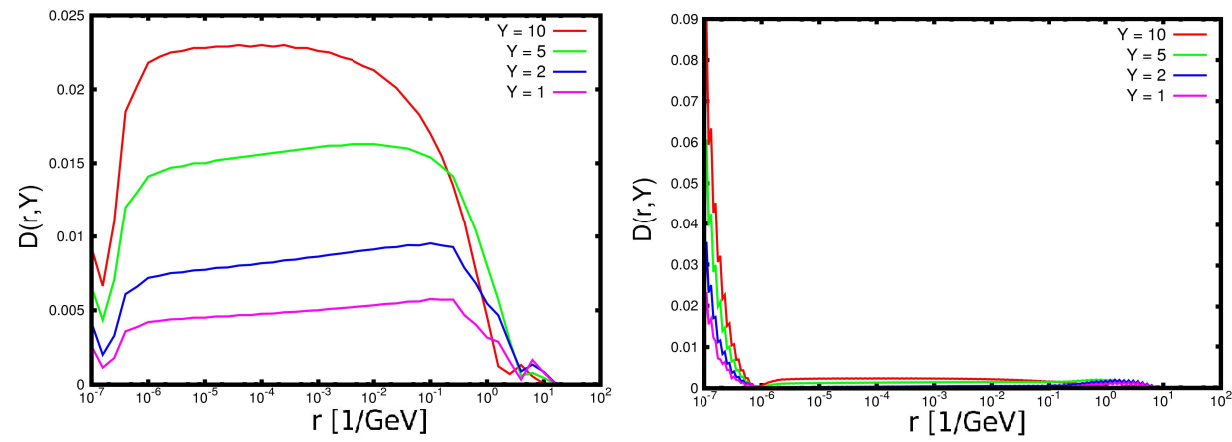

Figure 4. A variation of the step in integration over $r$, left - the step of 10 over one order of magnitude of $r_{1}$ compared to 25 , right - the step of 25 compared to the step of 50 .
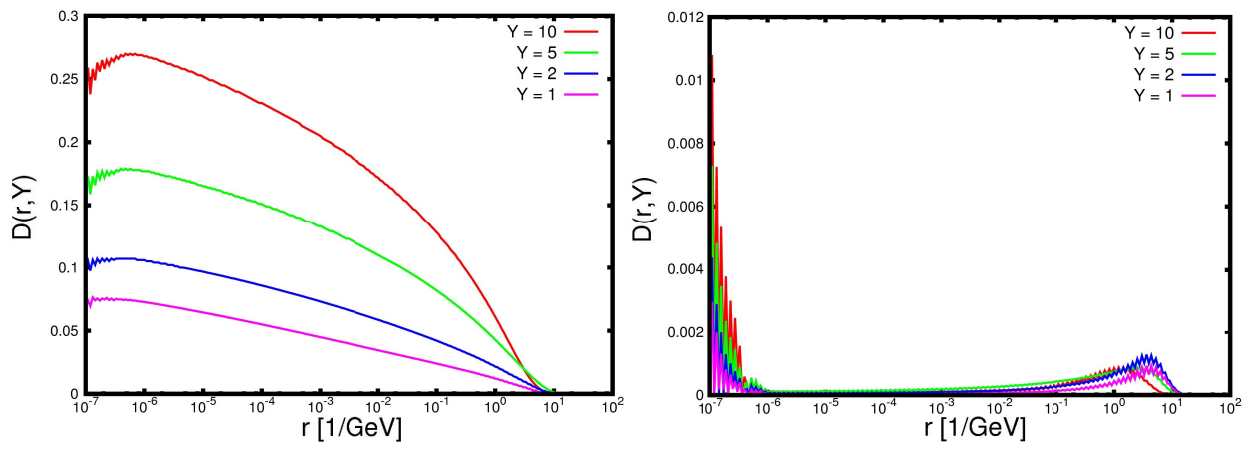

Figure 5. A variation of the step in integration over $\theta$. Left - the step of 5 over the interval of $[0, \pi]$ compared to 10 , right - the step of 10 compared to the step of 20.

Also, the variation of the number of steps for the angle $\theta$ between $\overrightarrow{r_{1}}$ and $\vec{r}$ from 5 steps to 10 steps over the interval $[0, \pi]$ has been performed resulting in huge difference up to $25 \%$ which quickly rises with rapidity. Adding more steps does not yield to any notable difference.

Running time for a fixed scale was about $100 \mathrm{~s}$ on a regular personal computer and the mean square variation was below $1.5 \%$ of the experimentally measured values of $F_{2}$.

\section{Conclusion}

There are several models that predict the effect of saturation of partons in high-energy collisions. Balitsky-Kovchegov evolution equation does that by modifying the BFKL equation with recombination processes that occur in hadrons at high energies.

The Balitsky-Kovchegov evolution equation was numerically solved using the Runge-Kutta method of fourth order, Simpson rule and linear interpolation. The parameters used for the numerical computation were tested and a suggestion for their setup has been put forward to obtain a good ratio of precision and computing speed since the program for solving the rcBK evolution equation can be very demanding on the running time. 
The optimal parameters to compute the rcBK equation prove to be:

- 25 steps per one order of magnitude of $r$,

- 10 steps in $\theta$ over the interval $[0 ; \pi]$,

- Runge-Kutta method of fourth order,

- A step of 0.01 in rapidity for the Runge-Kutta method.

\section{Acknowledgment}

This work was partially supported by the grant of the Grant Agency of Czech Republic n.13-20841S and by grant LK11209 of MŠMT ČR.

\section{References}

[1] V.S. Fadin, E.A. Kuraev, L.N. Lipatov, Phys. Lett. B60, 50 (1975)

[2] L.N. Lipatov, Sov. J. Nucl. Phys. 23, 338 (1976), [Yad. Fiz.23,642(1976)]

[3] E.A. Kuraev, L.N. Lipatov, V.S. Fadin, Sov. Phys. JETP 44, 443 (1976), [Zh. Eksp. Teor. Fiz.71,840(1976)]

[4] E. Kuraev, L. Lipatov, V.S. Fadin, Sov.Phys.JETP 45, 199 (1977)

[5] I. Balitsky, L. Lipatov, Sov.J.Nucl.Phys. 28, 822 (1978)

[6] I. Balitsky, Nucl. Phys. B463, 99 (1996), hep-ph/9509348

[7] Y.V. Kovchegov, Phys. Rev. D60, 034008 (1999), hep-ph/9901281

[8] J.L. Albacete, Y.V. Kovchegov, Phys. Rev. D75, 125021 (2007), 0704 . 0612

[9] J.L. Albacete, N. Armesto, J.G. Milhano, C.A. Salgado, Phys. Rev. D80, 034031 (2009), 0902.1112

[10] J.L. Albacete, N. Armesto, J.G. Milhano, P. Quiroga-Arias, C.A. Salgado, Eur. Phys. J. C71, 1705 (2011), 1012.4408

[11] M. Kuhlen, Springer Tracts Mod. Phys. 150, 1 (1999)

[12] Y.V. Kovchegov, Phys. Rev. D61, 074018 (2000), hep-ph/9905214

[13] J. Cepila, J.G. Contreras (2015), 1501.06687

[14] I. Balitsky, Phys. Rev. D75, 014001 (2007), hep-ph/0609105

[15] Y.V. Kovchegov, H. Weigert, Nucl. Phys. A784, 188 (2007), hep-ph/0609090

[16] J.L. Albacete, C. Marquet, Prog. Part. Nucl. Phys. 76, 1 (2014), 1401.4866

[17] F.D. Aaron et al. (ZEUS, H1), JHEP 01, 109 (2010), 0911.0884 